\title{
Reproducibility of noninvasive cardiac output during arm exercise in spinal cord injury
}

\author{
Jonathan Myers, PhD; ${ }^{1-2 *}$ Heather Brown, PT; ${ }^{3}$ Susan Smith, PT; ${ }^{3}$ Inder Perkash, MD; ${ }^{3}$ B. Jenny Kiratli, PhD \\ ${ }^{1}$ Cardiology Division, Department of Veterans Affairs (VA) Palo Alto Health Care System, Palo Alto, CA; ${ }^{2}$ Cardiology \\ Department, Stanford University, Palo Alto, CA; ${ }^{3}$ Spinal Cord Injury Center, VA Palo Alto Health Care System, Palo \\ Alto, CA
}

\begin{abstract}
The carbon dioxide $\left(\mathrm{CO}_{2}\right)$ rebreathing method is a noninvasive technique to estimate cardiac output during exercise, but few data are available on the validity and reliability of this measure in individuals with spinal cord injury (SCI). Sixteen male subjects with SCI (mean age $45+/-9$, seven paraplegic and nine tetraplegic) underwent three submaximal steady state arm ergometer exercise tests. We estimated cardiac output using the exponential $\mathrm{CO}_{2}$ rebreathing technique at an individualized exercise intensity approximating $50 \%$ of peak oxygen uptake. Mean values for the cardiac output measurements were $13.0+/-2.4,13.3+/-2.0$, and $13.4+/-1.7 \mathrm{~L} / \mathrm{min}$; the difference among the trials was not significant $(p=0.54)$. The typical error was $1.80+/-0.85 \mathrm{~L} / \mathrm{min}$, the limits of agreement were 11.3 to $15.3 \mathrm{~L} / \mathrm{min}$, the coefficient of variation was $5.4 \%+/-$ $3.4 \%$, and the intraclass correlation coefficient was 0.85 (95\% confidence interval $=0.70-0.94)$. The test-to-test variation in estimated cardiac output during arm ergometry in individuals with SCI is similar to that observed in studies that used this technique in ambulatory persons. The 5\% relative variation between tests suggests that the $\mathrm{CO}_{2}$ rebreathing technique for estimating cardiac output can be performed in SCI individuals with acceptable reproducibility.
\end{abstract}

Key words: activity, autonomic function, cardiac output, exercise testing, heart disease, heart rate, paraplegia, oxygen uptake, reproducibility, spinal cord injury.

\section{INTRODUCTION}

Advances in the medical management of spinal cord injury (SCI) over the last 30 years have resulted in a shift in the leading causes of death in these individuals from infections (e.g., pneumonia) to cardiovascular disease. Today, cardiovascular mortality in SCI approximates that of the nondisabled population [1]. One factor that likely contributes to high cardiovascular mortality in SCI is the inordinately low level of physical activity in these individuals [2-5]. Low physical activity levels in SCI have been attributed not only to the relatively small muscle mass available to perform work but also to societal factors, accessibility to facilities, transportation, and other factors [2-5].

In light of the high cardiovascular morbidity and mortality in SCI and the high prevalence of physical inactivity, efforts have been made to promote physical activity in SCI. In this context, methods designed to measure changes in cardiovascular performance associated with physical activity are also important. The most fundamental measure of cardiovascular performance is cardiac output; any improvement in fitness (measured by peak oxygen uptake $\left[\mathrm{VO}_{2}\right]$ ) is usually paralleled by an increase

\footnotetext{
Abbreviations: ANOVA $=$ analysis of variance, ICC $=$ intraclass correlation coefficient, $\mathrm{SCI}=$ spinal cord injury, $\mathrm{SD}=$ standard deviation, $\mathrm{VCO}_{2}=$ rate of $\mathrm{CO}_{2}$ production, $\mathrm{VO}_{2}=$ oxygen uptake.

* Address all correspondence to Jonathan Myers, PhD; VA Palo Alto Health Care System, Cardiology 111C, 3801 Miranda Ave, Palo Alto, CA 94304; 650-493-5000, ext 64661; fax: 650-852-3473. Email: drj993@aol.com
}

DOI: 10.1682/JRRD.2006.08.0103 
in cardiac output. Because the direct measurement of cardiac output requires an invasive procedure and therefore is expensive and carries added risk, noninvasive estimates of cardiac output are more frequently used. One such method involves individuals rebreathing a mixture of carbon dioxide $\left(\mathrm{CO}_{2}\right)$ so that venous $\mathrm{CO}_{2}$ content can be estimated and used to complete the Fick equation [6-8].

Studies in nondisabled subjects have demonstrated that this method is reasonably accurate when compared with invasive methods (direct Fick or thermodilution) or other noninvasive techniques [6-12]. Most investigators have observed high day-to-day reproducibility of cardiac output measured by $\mathrm{CO}_{2}$ rebreathing [13-14], and others have documented the procedure's validity by comparing it with cardiac output estimated from published regression lines relating to $\mathrm{VO}_{2}$ [15-16]. However, few data are available on employing the $\mathrm{CO}_{2}$ rebreathing technique in individuals with SCI, and to our knowledge no studies have assessed the reproducibility of this technique in an SCI population. The present study evaluated the reproducibility of cardiac output estimated during submaximal exercise by the $\mathrm{CO}_{2}$ rebreathing technique among individuals with SCI.

\section{METHODS}

\section{Participants}

Sixteen male subjects with SCI participated in the study (mean \pm standard deviation [SD] age $44.0 \pm 9.4$ ) (Table 1). Seven of the subjects were paraplegic and nine were tetraplegic; the mean \pm SD duration of injury was $17.0 \pm 9.4$ years. Seven of the subjects with tetraplegia had complete injuries and all the injuries in the subjects with paraplegia were complete. All subjects were free of cardiovascular disease and had adequate strength and motor function to perform a maximal arm ergometry protocol. Activity status was classified as "sedentary" if the subject reported engaging in three sessions a week of moderate activity lasting a minimum of $30 \mathrm{~min}$. Written informed consent was obtained from all subjects with a protocol approved by the Stanford University Investigational Review Board.

Table 1.

Demographic information.

\begin{tabular}{|c|c|c|c|c|c|c|c|}
\hline Subjects & Age & $\begin{array}{l}\text { Activity } \\
\text { Level }^{*}\end{array}$ & $\begin{array}{c}\text { Weight } \\
\text { (kg) }\end{array}$ & $\begin{array}{l}\text { Level of } \\
\text { Injury }\end{array}$ & $\begin{array}{c}\text { Completeness of } \\
\text { Injury }\end{array}$ & $\begin{array}{l}\text { Duration of } \\
\text { Injury (yr) }\end{array}$ & $\begin{array}{l}\text { Peak VO } \\
(\mathrm{mL} / \mathrm{min}) \\
\end{array}$ \\
\hline \multicolumn{8}{|l|}{ Tetraplegia } \\
\hline A & 38 & 0 & 68.2 & C6 & Complete & 21 & 784.3 \\
\hline $\mathrm{C}$ & 44 & 1 & 81.4 & C5-6 & Complete & 23 & 919.8 \\
\hline $\mathrm{D}$ & 53 & 1 & 94.6 & C6-7 & Complete & 13 & 614.9 \\
\hline $\mathrm{G}$ & 55 & 1 & 69.7 & C5-8 & Complete & 32 & 578.7 \\
\hline $\mathrm{H}$ & 49 & 0 & 58.3 & C6-7 & Complete & 17 & 548.0 \\
\hline I & 44 & 0 & 63.4 & C6 & Complete & 16 & 627.7 \\
\hline$\overline{\text { Mean } \pm \text { SD }}$ & $44.2 \pm 7.5$ & - & $70.7 \pm 12.7$ & - & - & $17.0 \pm 8.0$ & $682.6 \pm 130$ \\
\hline M & 47 & 0 & 124.9 & $\mathrm{~T} 4$ & Complete & 11 & $1,274.0$ \\
\hline $\mathrm{N}$ & 28 & 1 & 59.8 & T9-12 & Complete & 8 & $1,453.1$ \\
\hline $\mathrm{O}$ & 29 & 1 & 73.1 & T4-T7 & Complete & 8 & $1,330.4$ \\
\hline $\mathrm{P}$ & 53 & 0 & 94.6 & T5 & Complete & 20 & 955.5 \\
\hline$\overline{\text { Mean } \pm \text { SD }}$ & $43.7 \pm 12.0$ & - & $81.3 \pm 21.9$ & - & - & $17.0 \pm 11.5$ & $1,229.4 \pm 260$ \\
\hline
\end{tabular}




\section{Exercise Testing}

Initially, all participants provided a complete medical history and underwent a physical examination and then performed a maximal exercise test using a manually incremented arm crank protocol. This test habituated subjects to the procedure and gas exchange apparatus, established clinical stability, and determined peak $\mathrm{VO}_{2}$. Cardiac output measurements were performed on a separate day. Subjects were asked to abstain from food, coffee, and smoking for at least 3 hours prior to testing. Testing was performed in the upright seated position with a Monark arm ergometer (Monark Excercise AB, Varberg, Sweden) mounted to an adjustable table. Subjects were asked to maintain the arm crank cadence at 60 rpm throughout the test. Changes in resistance of the arm ergometer were individualized (increments ranging from $1-5 \mathrm{~W} / \mathrm{min}$ ) to yield a test duration of approximately 8 to $12 \mathrm{~min}$. A standard 12-lead encephalogram was obtained throughout the exercise test and recovery period. Blood pressure was measured before and immediately after the exercise test. Exercise was continued until subjects exhibited volitional fatigue, and the Borg 6-20 scale was used to quantify subjective effort [17]. The test was terminated when the subject was no longer able to maintain $50 \mathrm{rpm}$ at a given workload.

\section{Cardiac Output}

On a separate day from the maximal exercise test, cardiac output was determined with the use of three separate submaximal arm ergometry trials. From the baseline exercise test, a submaximal work rate was chosen for each subject to approximate 50 percent of maximal exercise capacity and a perceived exertion level of approximately 10 to 12 (Borg 6-20 scale). Cardiac output was measured during submaximal exercise with a $\mathrm{CO}_{2}$ rebreathing technique developed by Defares [18] and described in detail elsewhere $[6-7,19]$. This technique is based on the application of $\mathrm{CO}_{2}$, rather than $\mathrm{O}_{2}$, to the Fick equation:

$$
\text { Cardiac Output }=\frac{\mathrm{VCO}_{2}}{\mathrm{a}-\mathrm{vCO}_{2} \text { difference }},
$$

where $\mathrm{VCO}_{2}$ is the rate of $\mathrm{CO}_{2}$ production and a-vCO difference is the difference in the $\mathrm{CO}_{2}$ content between the arterial and venous blood. Arterial $\mathrm{CO}_{2}$ content was estimated from end-tidal partial pressure of $\mathrm{CO}_{2}$ from the metabolic system (Medical Graphics Corp., St. Paul, Minnesota). We determined mixed venous $\mathrm{CO}_{2}$ content by having participants rebreath a $\mathrm{CO}_{2}$ gas mixture and estimating an equilibrium point between the $\mathrm{CO}_{2}$ content of the lung and the venous blood.

Subjects performed arm ergometry at their respective workloads until a constant $\mathrm{VO}_{2}$ was achieved (approximately $4 \mathrm{~min}$ ). They then began rebreathing a 4 percent $\mathrm{CO}_{2} / 35$ percent $\mathrm{O}_{2}$ (balance nitrogen) gas mixture for a period of approximately $20 \mathrm{~s}$. An exponential curve for the rise in $\mathrm{VCO}_{2}$ was generated, representing the point at which the $\mathrm{CO}_{2}$ content of the lung was equal to that of the mixed venous blood. This value for venous $\mathrm{CO}_{2}$ content completes the Fick equation, permitting an estimation of cardiac output. This procedure was repeated three times for each subject; a 5 to 10 min period between trials was given for subjects to rest and to permit complete washout of $\mathrm{CO}_{2}$ from the circulation.

\section{Data Analysis}

Mean differences in cardiac output measured across the three trials were assessed by repeated measures analysis of variance (ANOVA). Reproducibility of cardiac output was assessed by the typical error, limits of agreement, coefficient of variation, and intraclass correlation coefficient (ICC). The ICC was calculated with the two-way mixed effect model. NCSS software (Kayesville, Utah) was used for all statistical analyses.

\section{RESULTS}

Mean \pm SD values from the maximal exercise tests are presented in Table 2. Peak perceived exertion was $19.3 \pm$ 1.0 , and peak respiratory exchange ratio was $1.31 \pm 0.16$;

Table 2.

Hemodynamic responses to maximal arm ergonometry exercise test.

\begin{tabular}{lc}
\hline \multicolumn{1}{c}{ Measurements } & Mean \pm SD \\
\hline Heart Rate (bpm) & $57 \pm 10$ \\
Systolic Blood Pressure (mmHg) & $128 \pm 17$ \\
Diastolic Blood Pressure (mmHg) & $81 \pm 10$ \\
Peak Exercise & \\
$\quad$ Heart Rate (bpm) & $134 \pm 31$ \\
Oxygen Uptake (mL/kg/min) & $12.6 \pm 4.9$ \\
Ventilation (L/min) & $48.0 \pm 18.5$ \\
Respiratory Exchange Ratio & $1.31 \pm 0.16$ \\
$\quad$ Rating of Perceived Exertion & $19.3 \pm 1.0$ \\
Cardiac Output (L/min) & $13.0 \pm 1.8$ \\
\hline SD = standard deviation.
\end{tabular}


these values suggest that subjects generally provided maximal efforts. The mean peak $\mathrm{VO}_{2}$ was $12.6 \pm 4.9 \mathrm{~mL} / \mathrm{kg} /$ min, and maximal heart rate was $134 \pm 31 \mathrm{bpm}$, values that are typical of heterogenous subjects with SCI $[4-5,20]$.

Results from the repeated cardiac output trials are presented in Table 3. Mean \pm SD values for the three cardiac output measurements were $13.0 \pm 2.4,13.3 \pm 2.0$, and $13.4 \pm 1.7 \mathrm{~L} / \mathrm{min}$, respectively. The ANOVA model did not detect a significant trend across the three trials $(p=0.54)$. The typical error was $1.80 \pm 0.85 \mathrm{~L} / \mathrm{min}$, the limits of agreement were 11.3 to $15.3 \mathrm{~L} / \mathrm{min}$, the coefficient of variation was $5.4 \pm 3.4$ percent, and the ICC was $0.85(95 \%$ confidence interval $=0.70-0.94)$.

\section{DISCUSSION}

Individuals with SCI exhibit deficient vasomotor control due to an interruption of the pathways linking the supraspinal centers and the lower-limb vasculature. The absence of both normal vasomotor tone and the lowerlimb muscle pump during activity restricts venous return and contributes to reductions in the cardiac output response to exercise in SCI [2-5]. To gain insight into the cardiovascular effects of various interventions in subjects with SCI, researchers have employed noninvasive measures of cardiac output responses to exercise in studies addressing the effects of functional electrical stimulation [21-25], passive leg exercise [26], orthostatic challenge [27], and exercise training [22-23]. The $\mathrm{CO}_{2}$ rebreathing technique has been used since the 1950s to estimate cardiac output during exercise in ambulatory individuals $[18,28]$, and studies have documented its reliability and reproducibility [6-10]. In theory, the smaller muscle mass employed during arm exercise in subjects with SCI could influence the recirculation of $\mathrm{CO}_{2}$ in the blood during rebreathing and therefore the rate of rise in $\mathrm{CO}_{2}$ content in the lung, thus affecting the accuracy of the extrapolation curve from which venous $\mathrm{CO}_{2}$ content is estimated. To our knowledge, no studies have addressed the reliability or reproducibility of this technique in subjects with SCI.
Acceptable reproducibility of a measure must be confirmed before it can be considered to have valid clinical application. While high reproducibility does not ensure accuracy, it quantifies the degree to which the test produces the same result each time it is conducted. Because the $\mathrm{CO}_{2}$ rebreathing technique only estimates cardiac output, it is commonly measured in three separate trials $[6-7,19,29]$. In the present study, we assessed reproducibility of these trials using several standardized measures of reliability: the typical error, coefficient of variation, ICC, and limits of agreement (Table 3). The typical error is an expression of the random variation across measurements for a given subject; it is defined simply as the SD of the three trials for that individual. The coefficient of variation is a measure of the relative variation, or variation relative to the size of the mean for the three measurements expressed as a percentage. The limits of agreement are, in effect, the 95 percent confidence limits within which any given measurement would fall. The ICC reflects the degree of association among the three trials.

In ambulatory individuals tested either on the treadmill or leg cycle ergometer, coefficients of variation for cardiac output measured with the rebreathing technique have been reported to range between 5 and 10 percent $[6,15-16,19]$, although one study observed coefficients of variation of 0.8 and 1.4 percent for workloads of 50 and $100 \mathrm{~W}$, respectively [16]. Thus, the coefficient of variation we observed (5.1\%) is typical of the values observed for ambulatory individuals using the $\mathrm{CO}_{2}$ rebreathing technique. This finding suggests that for a given measurement of cardiac output by $\mathrm{CO}_{2}$ rebreathing in an individual with SCI, the value can be expected to vary by about 5 percent from trial to trial for that individual. Importantly, this falls within the range of coefficients of variation observed for more direct measurements of cardiac output, i.e., invasive determination by the Fick or thermodilution dye techniques [6,19]. Our observed limits of agreement (11.3-15.3 L/min) indicate that a subsequent trial in any given participant would fall within these limits 95 percent of the time. The typical error, $1.80 \mathrm{~L} / \mathrm{min}$,

Table 3.

Reproducibility of cardiac output measurements (mean \pm standard deviation).

\begin{tabular}{|c|c|c|c|c|c|c|c|c|}
\hline Measurement & Test 1 & Test 2 & Test 3 & Mean & $\begin{array}{l}\text { Typical } \\
\text { Error }\end{array}$ & $\begin{array}{c}\text { Limits of } \\
\text { Agreement }\end{array}$ & $\begin{array}{c}\text { ICC } \\
(95 \% \text { CI })\end{array}$ & $\begin{array}{l}\text { Coefficient of } \\
\text { Variation (\%) }\end{array}$ \\
\hline Cardiac Output (L/min) & $13.0 \pm 2.4$ & $13.3 \pm 2.0$ & $13.4 \pm 1.7$ & $13.3 \pm 2.0$ & $1.80 \pm 0.85$ & $11.3-15.3$ & $0.85(0.70-0.94)$ & $5.4 \pm 3.4$ \\
\hline
\end{tabular}


represents the variation we would expect to see from trial to trial if any one of our participants performed multiple trials. Finally, the ICC we observed (0.85) suggests that a strong association existed among the repeated measures.

The differences in coefficients of variation for repeated measurements of noninvasive cardiac output in previous studies are likely due to differences in exercise intensity used (for example, a stable steady state maybe more difficult to achieve at higher workloads) but may also be attributable to differences in the $\mathrm{CO}_{2}$ rebreathing method used (equilibrium or exponential). We used the exponential technique based on the early observations of Clausen et al. [30], who performed repeated measures of cardiac output using the equilibrium method and suggested that the reproducibility of this particular method was unacceptable for use during exercise. Given the 5 to 7 percent coefficients of variation for cardiac output reported using the direct Fick technique (considered the gold standard), the reproducibility of the indirect cardiac output measurements that we observed appears to fall within the range of biologic variability. This result suggests that this method of determining cardiac output noninvasively during submaximal exercise provides acceptable reliability for assessing the cardiovascular response to exercise in individuals with SCI.

Our study was limited to the assessment of reproducibility; we did not address the accuracy of the technique. An assessment of accuracy would require comparison to the direct Fick method, necessitating an invasive sample of mixed venous $\mathrm{CO}_{2}$ content (requiring catheterization of the right side of the heart), a measurement that would be impractical to obtain among SCI individuals performing upper-body exercise. In addition, we studied only one method of estimating cardiac output, $\mathrm{CO}_{2}$ rebreathing using the exponential rise in $\mathrm{VCO}_{2}$ to estimate the $\mathrm{CO}_{2}$ content of the mixed venous blood. Our results may have been different had we employed the alternative method, $\mathrm{CO}_{2}$ rebreathing using the equilibrium technique to estimate mixed venous $\mathrm{CO}_{2}$ or newer methods such as acetylene uptake [31] or bioimpedance [32]. Finally, the heterogeneity of the sample may have artificially raised the ICC, and the results may differ in a more homogeneous group of subjects.

\section{CONCLUSIONS}

Cardiac output is the fundamental measure of cardiovascular function; a noninvasive technique that is accurate and reproducible has obvious applications for assessing therapeutic interventions not only in cardiovascular disease but also for a wide variety of chronic diseases and disabilities. Although the literature is replete with studies on the reliability and reproducibility of the noninvasive $\mathrm{CO}_{2}$ rebreathing technique in ambulatory individuals, such studies have not been performed among individuals with SCI. The high retest correlation, low typical error, and high coefficient of variation observed in our study suggest that the $\mathrm{CO}_{2}$ rebreathing technique for estimating cardiac output can be performed among SCI individuals with acceptable reproducibility. These findings have applications for the growing body of literature assessing cardiovascular responses to functional electrical stimulation, exercise training, or other interventions among individuals with SCI.

\section{ACKNOWLEDGMENTS}

This material was based on work supported in part by the Department of Veterans Affairs, Rehabilitation Research and Development Service, grant B1629-RA.

The authors have declared that no competing interests exist.

\section{REFERENCES}

1. Garshick E, Kelley A, Cohen SA, Garrison A, Tun CG, Gagnon D, Brown R. A prospective assessment of mortality in chronic spinal cord injury. Spinal Cord. 2005;43(7): 408-16. [PMID: 15711609]

2. Myers J, Lee M, Kiratli B. Cardiovascular disease in spinal cord injury: An overview of prevalence, risk, evaluation, and management. Am J Phys Med Rehabil. 2007;86(2): 142-52. [PMID: 17251696]

3. Washburn RA, Figoni SF. Physical activity and chronic cardiovascular disease prevention in spinal cord injury: A comprehensive literature review. Top Spinal Cord Inj Rehabil. 1998;3(3):16-32.

4. Jacobs PL, Nash MS. Exercise recommendations for individuals with spinal cord injury. Sports Med. 2004;34(11): 727-51. [PMID: 15456347]

5. Phillips WT, Kiratli BJ, Sarkarati M, Weraarchakul G, Myers J, Franklin BA, Parkash I, Froelicher V. Effect of spinal cord injury on the heart and cardiovascular fitness. Curr Probl Cardiology. 1998;23(11):641-716. [PMID: 9830574]

6. Kirby TE. The $\mathrm{CO}_{2}$ rebreathing technique for determination of cardiac output: Part I. J Cardiovasc Rehabil Prev. 1985;5:97-101. 
7. Kirby TE. The $\mathrm{CO}_{2}$ rebreathing technique for determination of cardiac output: Part II. J Cardiovasc Rehabil Prev. 1985;5:132-38.

8. Olszowka AJ, Shykoff BE, Pendergast DR, Lundgren CE, Farhi LE. Cardiac output: A view from Buffalo. Eur J Appl Physiol. 2003;90(3-4):292-304. [PMID: 12942332]

9. Beekman RH, Katch V, Marks C, Rocchini AP. Validity of $\mathrm{CO}_{2}$ rebreathing cardiac output during rest and exercise in young adults. Med Sci Sports Exerc. 1984;16(3):306-10. [PMID: 6431221]

10. Wilmore JH, Farrell PA, Norton AC, Cote RW, Coyle EF, Ewy GA, Temkin LP, Billing JE. An automated, indirect assessment of cardiac output during rest and exercise. J Appl Physiol. 1982;52(6):1493-97. [PMID: 7107459]

11. Christensen TB, Jensen BV, Hjerpe J, Kanstrup IL. Cardiac output measured by electric bioimpedance compared with the $\mathrm{CO}_{2}$ rebreathing technique at different exercise levels. Clin Physiol. 2000;20(2):101-5. [PMID: 10735976]

12. Pianosi PT. Impedance cardiography accurately measures cardiac output during exercise in children with cystic fibrosis. Chest. 1997;111(2):333-37. [PMID: 9041978]

13. Vanhees L, Defoor J, Schepers D, Brusselle S, Reybrouck T, Fagard R. Comparison of cardiac output measured by two automatic methods of $\mathrm{CO}_{2}$ rebreathing. Med Sci Sports Exerc. 2000;32(5):1028-34. [PMID: 10795797]

14. Nilsson LB, Eldrup N, Berthelsen PG. Lack of agreement between thermodilution and carbon dioxide-rebreathing cardiac output. Acta Anaesthesiol Scand. 2001;45(6):680-85. [PMID: 11421824]

15. Zeidifard E, Silverman M, Godfrey S. Reproducibility of the indirect $\left(\mathrm{CO}_{2}\right)$ Fick method for calculation of cardiac output. J Appl Physiol. 1972;33(1):141-43. [PMID: 5037399]

16. Knowlton RG, Adams GE. The consistency of carbon dioxide rebreathing as a non-invasive method to determine exercise cardiac output. Ergonomics. 1974;17(2):241-48. [PMID: 4845891]

17. Borg G. Borg's perceived exertion and pain scales. Champaign (IL): Human Kinetics; 1998.

18. Defares J. Determination of $\mathrm{PVCO}_{2}$ from the exponential $\mathrm{CO}_{2}$ rise during rebreathing. J Appl Physiol. 1958;13(2): 159-64. [PMID: 13575322]

19. Heigenhauser GJ, Jones NL. Measurement of cardiac output by carbon dioxide rebreathing methods. Clin Chest Med. 1989;10(2):255-64. [PMID: 2500297]

20. Hayes AM, Myers JN, Ho M, Lee MY, Perkash I, Kiratli BJ. Heart rate as a predictor of energy expenditure in people with spinal cord injury. J Rehabil Res Dev. 2005;42(5): 617-24. [PMID: 16586187]

21. Taylor PN, Ewins DJ, Fox B, Grundy D, Swain ID. Limb blood flow, cardiac output and quadriceps muscle bulk following spinal cord injury and the effect of training for the Odstock functional electrical stimulation standing system. Paraplegia. 1993;31(5):303-10. [PMID: 8332376]
22. Faghri PD, Glaser RM, Figoni SF. Functional electrical stimulation leg cycle ergometer exercise: Training effects on cardiorespiratory responses of spinal cord injured subjects at rest and during submaximal exercise. Arch Phys Med Rehabil. 1992;73(11):1085-93. [PMID: 1444777]

23. Hooker SP, Figoni SF, Rodgers MM, Glaser RM, Mathews T, Suryaprasad AG, Gupta SC. Physiologic effects of electrical stimulation leg cycle exercise training in spinal cord injured persons. Arch Phys Med Rehabil. 1992;73(5):470-76. [PMID: 1580776]

24. Davis GM, Servedio FJ, Glaser RM, Gupta SC, Suryaprasad AG. Cardiovascular responses to arm cranking and FNSinduced leg exercise in paraplegics. J Appl Physiol. 1990; 69(2):671-77. [PMID: 2228881]

25. Raymond J, Davis GM, Climstein M, Sutton JR. Cardiorespiratory responses to arm cranking and electrical stimulation leg cycling in people with paraplegia. Med Sci Sports Exerc. 1999;31(6):822-28. [PMID: 10378909]

26. Muraki S, Yamasaki M, Ehara Y, Kikuchi K, Seki K. Cardiovascular and respiratory responses to passive leg cycle exercise in people with spinal cord injuries. Eur J Appl Physiol Occup Physiol. 1996;74(1-2):23-28. [PMID: 8891496]

27. Raymond J, Davis GM, Bryant G, Clarke J. Cardiovascular responses to an orthostatic challenge and electricalstimulation-induced leg muscle contractions in individuals with paraplegia. Eur J Appl Physiol Occup Physiol. 1999; 80(3):205-12. [PMID: 10453922]

28. Collier CR. Determination of mixed venous $\mathrm{CO}_{2}$ tensions by rebreathing. J Appl Physiol. 1956;9(1):25-29. [PMID: 13357408$]$

29. Atwood JE, Myers J, Quaglietti S, Grumet J, Gianrossi R, Umman T. Effect of betaxolol on the hemodynamic, gas exchange, and cardiac output response to exercise in chronic atrial fibrillation. Chest. 1999;115(4):1175-80. [PMID: 10208225]

30. Clausen JP, Larsen OA, Trap-Jensen J. Cardiac output in middle-aged patients with $\mathrm{CO}_{2}$ rebreathing method. J Appl Physiol. 1970;28(3):337-42. [PMID: 4905800]

31. Dibski DW, Smith DJ, Jensen R, Norris SR, Ford GT. Comparison and reliability of two non-invasive acetylene uptake techniques for the measurement of cardiac output. Eur J Appl Physiol. 2005;94(5-6):670-80. [PMID: 15918060]

32. Moshkovitz Y, Kaluski E, Milo O, Vered Z, Cotter G. Recent developments in cardiac output determination by bioimpedance: Comparison with invasive cardiac output and potential cardiovascular applications. Curr Opin Cardiol. 2004;19(3):229-37. [PMID: 15096956]

Submitted for publication August 29, 2006. Accepted in revised form March 2, 2007. 